ISSN: 0210-1696

DOI: http://dx.doi.org/10.14201/scero20154644558

\title{
VALORACIÓN DE UNA EXPERIENCIA DE EDUCACIÓN AFECTIVO-SEXUAL PARA PERSONAS CON DISCAPACIDAD INTELECTUAL
}

\section{Assessment of Emotional-Sexual Education Experience for People with Intellectual Disabilities}

\author{
Leticia Vizcaino Luque \\ Máster en Psicología de la Educación. Universidad de La Laguna
}

Ramón Aciego de Mendoza Lugo

Psicología Evolutiva y de la Educación. Facultad de Ciencias de la Salud. Universidad de La Laguna. Campus de Guajara. 38071. La Laguna. Tenerife. Islas Canarias

raciego@ull.es

Recepción: 12 de junio de 2015

Fecha de aceptación definitiva: 20 de octubre de 2015

Biblid. [0210-1696 (2015) vol. 46 (4), n. ${ }^{\circ} 256$, octubre-diciembre; 45-58]

Resumen: Se realizó esta experiencia como respuesta a la demanda de un Centro de Educación Especial de Tenerife (Canarias-España) para el desarrollo de una acción formativa afectivo-sexual. La intervención se lleva a cabo en dos grupos. Uno, constituido por ocho usuarios del Centro Escolar, de entre 16-22 años, y otro por siete usuarios del Centro Ocupacional, de entre 22-39 años. Se diseña una intervención tomando como referencia las Carpetas didácticas de educación afectivo-sexual (Bolaños, González, Jiménez, Ramos-Rodríguez y Rodríguez, 1994). Se realiza una valoración a tres niveles (usuarios, educadores y familias), antes y después de la intervención. En cuanto a la evaluación de línea base, los tres agentes detectaron niveles similares en todas las áreas de intervención, si bien en el área sexualidad señalan ciertas lagunas en el conocimiento y destreza de diferentes prácticas erótico-sexuales. Los contrastes entre la evaluación prepost arrojaron diferencias significativas en afectividad, habilidades sociales y sexualidad. Se considera que, tras la intervención, los usuarios tienen mayor conocimiento sobre prácticas sexuales y afectivas; y diferencian entre contextos (privado/público) y relaciones (amigos, conocidos y desconocidos). 
Palabras Clave: intervención psicoeducativa; discapacidad intelectual; educación especial; educación afectivo-sexual.

Aвstract: This article responds to the demand for a Special Education Center on the island of Tenerife (Canary Islands - Spain) to develop training on emotional health and sexuality. The intervention is carried out in two groups. One, consisting of eight members of the Special School of 16-22 years, and other with seven other members of the Occupational Center of between 22-39 years. Intervention is designed with reference to the folders of affective and sex education (Bolaños González Jiménez, Ramos-Rodriguez and Rodriguez, 1994). The evaluation was realized in three levels (users, educators and families), before and after the intervention. Regarding the baseline assessment, these three groups have detected similar levels in all areas of intervention, although they indicated some gaps in the knowledge and skill of different erotic-sex practices. Through the contrasts between pre and post-evaluation significant effects are evident in affective, social skills and sexual areas. That means that users, educators and families believe that after-intervention, users have greater knowledge about the manifestations of affection, enhance positive samples (hugging, touching, etc.) and differentiate between contexts (private / public) and relationships (friends, acquaintances and strangers).

KEYS wORDS: psychoeducational intervention; intellectual disabilities; special education; sexual-affective education.

\section{Introducción}

$\mathrm{E}$

XISTEN DIVERSOS PROGRAMAS DESTINADOS A FAVORECER la educación afectivosexual, pero resulta difícil encontrar programas adaptados a la población con discapacidad intelectual (Navarro, Torrico y López, 2010). El programa afectivo-sexual diseñado para esta intervención viene a ser una adaptación para estas personas del expuesto en las Carpetas didácticas de educación afectivo-sexual (Bolaños, González, Jiménez, Ramos-Rodríguez y Rodríguez, 1994; Colectivo Harimaguada, 2007), considerado como uno de los programas de referencia y de mayor implementación en Canarias (España), comunidad donde se desarrolla esta experiencia.

Conviene recordar que el término "discapacidad intelectual” hace referencia a las limitaciones que se producen antes de los 18 años tanto a nivel intelectual como en habilidades sociales, comunicativas, académicas, funcionales, laborales y de autorregulación (García-Alonso, 2005).

Existen diversas metodologías de trabajo que resultan claves para trabajar con esta población. Por una parte, se resalta la importancia de una educación emocional a través de tres dimensiones (la cognitiva, la afectiva y la conductual). En ésta, el aula/taller es un entorno de convivencia que permite que el proceso de enseñanzaaprendizaje se realice mediante dinámicas grupales (Rubio, 1995). Por otra parte, las técnicas de expresión corporal persiguen el aprendizaje de la expresión-coordinación corporal y la comunicación "no verbal", favoreciendo un desarrollo emocional, psicofisiológico y social equilibrado (Warrick, 2002). Entre estas técnicas encontramos: 
la actividad física y manipulativa, la musicoterapia, la risoterapia, la psicomotricidad y la expresión creativa en sus diferentes vertientes (plástica, musical, corporal, artística...) (Alonso y Aciego, 2003).

A la hora de diseñar un programa orientado a satisfacer la educación "afectivo-sexual” es necesario considerar este ámbito como una necesidad innata, universal y educable, como se propone en la Ley Orgánica de Educación (LOE 2/2006, de 3 de mayo).

Asimismo, es importante dilucidar algunas creencias erróneas sobre las personas con discapacidad, arraigadas en la población normalizada. Como, por ejemplo, la creencia de que por el propio hecho de educar se despierta el deseo sexual y/o el considerarles como seres asexuados o sexualmente infantiles y que, por lo tanto, no existe motivo para formarles y educarles. Concepciones que llevan a afirmar que las conductas afectivo-sexuales inadecuadas de estas personas no están tanto asociadas a la discapacidad como a la falta de un proceso continuo de formación, dato que justifica la necesidad de esta intervención (Rodríguez-Mayoral, Moretin, Arias y López, 2006).

Como cierre de este apartado, remarquemos algunas de las pautas que se han de tener en consideración a la hora de implementar acciones de educación afectivo-sexual con personas con discapacidad intelectual: ser coherentes con la perspectiva actual de la discapacidad; asumir un enfoque positivo de la sexualidad; aportar un enfoque multifocal en el que se destaque la implicación de familias y educadores; y desplegar una planificación estructurada y sistemática adaptando la metodología al grupo con el que se trabaja (Moretin, Rodríguez-Mayoral, Arias y Aguado, 2006; Moretin, VerdugoAlonso, Arias y Rodríguez-Mayoral, 2006).

\section{Propuesta de intervención}

\section{Participantes}

La intervención se dirigió a dos grupos de personas con discapacidad intelectual. El primer grupo lo constituyen 8 personas, 5 chicos y 3 chicas de entre 16 a 21 años, que forman parte de los 85 alumnos y alumnas del Centro Escolar. Dicho Centro Escolar cuenta con 12 aulas, 3 de educación primaria y el resto, de tránsito a la vida adulta. El segundo grupo quedó compuesto por 7 personas, 3 hombres y 4 mujeres de entre 22 a 39 años, que acuden al Centro Ocupacional en jornada de 9 a 14 horas. En éste, se encuentran trabajando 60 personas que realizan diferentes tareas manuales para su posterior venta.

\section{Metodología}

\section{Contenidos}

El programa se diseñó basándose en las Carpetas didácticas de educación afectivosexual (Bolaños, González, Jiménez, Ramos-Rodríguez y Rodríguez, 1994; Colectivo Harimaguada, 2007). 
Dicho programa persigue satisfacer la educación afectivo-sexual de la población normalizada y se estructura en seis áreas principales. La primera es el área "corporal”, que abarca tanto las partes del cuerpo como los cambios de éste y la expresión corporal. La segunda es el área "social”, que incluye la escucha activa, la asertividad, la empatía, el respeto y la cohesión grupal. La tercera es el área "emocional”, en la que se trabajan las habilidades y destrezas relacionales como el autocontrol, reconocimiento y expresión de emociones, autoestima, etc. La cuarta es el área "afectivo-sexual”, que se centra en reconocer las muestras positivas de afecto, desdramatizando así el placer corporal. La quinta es la referida al "contexto privado/público", en la que se toma consciencia de la diferencia entre espacios públicos y espacios de intimidad. Por último, en el área de "maltrato o abuso sexual", se distingue entre contacto cómodo y abusivo, a la vez que se aprende a decir "no" y cómo actuar ante un caso de este tipo.

\section{Sesiones de intervención}

El periodo de observación llevado a cabo durante el primer trimestre (octubrediciembre) resultó fundamental para conocer las características y las motivaciones específicas de las personas que acuden al taller, y adaptar el programa a éstos. Se decidió trabajar las áreas de: "Cuerpo", "Inteligencia Emocional”, "Habilidades Sociales", "Afectividad", "Sexualidad" y "Abuso". Para cada una de éstas, se diseñaron entre dos o tres sesiones grupales. La intervención se llevó a cabo durante cuatro meses (febrero-mayo), en los que los usuarios acudieron a dos sesiones semanales de 60 minutos, dentro del horario del centro.

Como idea cardinal, hay que tener presente que el conocimiento no es la mera acumulación de información ni tampoco el resultado de la comprensión súbita de las explicaciones (Barragán, 1994). Por ello, la metodología utilizada se enmarca dentro de un enfoque constructivista en el que se parte de los conocimientos previos y motivaciones del grupo, construyendo el conocimiento por ellos mismos y adquiriendo e interiorizando destrezas y actitudes. El monitor/a sólo ayuda a que esta construcción se dé, guiando, apoyando y supervisando el proceso.

Se trata de una población con déficit cognitivo, por lo que el trabajo se adecuó a las posibilidades del grupo mediante una metodología activa y participativa (acorde con el constructivismo), vivencial, lúdica e integradora, intentando aunar distintas capacidades, competencias y destrezas buscando un aprendizaje significativo. Para ello se trabajaron habilidades y capacidades como la expresión y sensibilidad corporal, la capacidad manipulativa, habilidades emocionales y sociales, la lectura y escritura, y habilidades y conocimientos propios de la sexualidad. En relación a este último punto, se trabajaron cuestiones como conocer la anatomía y fisiología de los genitales, cambios físicos a lo largo del desarrollo, relación con el propio cuerpo, cuerpo como fuente de sensaciones placenteras, diferencias entre sexos, respeto hacia las demás personas y sus deseos, expresar sentimientos, manifestaciones afectivas, conductas de riesgo, medios anticonceptivos, masturbación, etc. Para llevar a cabo esta tarea, se utilizaron materiales como revistas, periódicos, tijeras, pegamento, colores, pintura 
de dedos, vendas o pañuelos, colchonetas, pelotas de baloncesto y de goma, sillas, etc., además de utilizar espacios como la clase, el salón de juegos y la cancha al aire libre.

A modo de ejemplo, se expone la dinámica de la sesión "Muchos peces pequeños pueden con el pez grande", incluida en el módulo de habilidades sociales y orientada a favorecer la cohesión grupal:

"Repasando lo aprendido": Para comenzar la sesión, se sentarán y hablarán sobre lo que recuerdan del día anterior durante 5-10 minutos, apoyándose en la "ficha”.

"Desarrollo":

- “Derecha-izquierda": Esta actividad ayudará a establecer el término "unión”. Para ello, deben colocarse uno detrás del otro, muy juntos, y formar un corro. A la señal, se sentarán en las piernas del compañero posterior y rodarán lentamente al ritmo que marca el monitor. Se jugará durante alrededor de 10 minutos.

- "Salvándonos del pez grande": Se distribuyen por el suelo 5 o 6 hojas grandes de periódico. Al ritmo de la música, deberán moverse y bailar por todo el espacio. Cuando la música pare, cada miembro deberá subirse encima de una hoja de periódico sin romperla y sin caerse de ésta. A medida que avance el juego, las hojas de periódico se irán haciendo más pequeñas, por lo que todos deberán ayudarse para no caer. Si alguien queda fuera, no puede moverse hasta que otro jugador le rescate. Para rescatar a un compañero será necesario darle un fuerte beso o abrazo. Se jugará durante 20-25 minutos.

¿Qué hemos aprendido?: A modo de resumen, se hará un mural (mar con olas). Dentro, habrá un pez de papel por cada uno de los miembros del grupo. Todos juntos, tendrán que pintar y decorar el mural y escribir una palabra sobre lo que aprendieron o dibujar una cara de cómo se lo pasaron en la sesión. En este caso, junto a la actividad de resumen se plantea la de relajación, estimulando la capacidad creativa mientras escuchan música relajante. Esto tendrá una duración de 10 minutos.

Como se puede observar a partir de este ejemplo, se mantiene una misma secuencia en todas las sesiones: repaso de la sesión anterior; desarrollo de actividades; relajación; y compromisos. Asimismo, se utiliza una metodología constructivista, activa y participativa, partiendo de sus conocimientos previos y motivaciones. Con ello, no sólo se favorece un clima lúdico e implicativo sino, también, se pretende aumentar las probabilidades de éxito en la adquisición de nuevas destrezas y actitudes. Estas actividades se realizan en el aula y en los espacios abiertos del centro (cancha, patio, etc.), utilizando diversos materiales como tijeras, revistas, pelotas, colchonetas, música, sus propios cuerpos, etc.

A continuación, se detalla la secuencia de las sesiones implementadas:

\section{Módulo de habilidades sociales}

Sesión 1. "Muchos peces pequeños pueden con el pez grande” (Cohesión)

Sesión 2. "Convirtiéndonos en superhéroes" (Escucha activa) 
Sesión 3. "Hablamos sin palabras” (Comunicación no-verbal)

Módulo de inteligencia emocional

Sesión 4. "Reconozco tus emociones y expreso las mías” (Emociones)

Sesión 5. "Me pongo en tu lugar” (Empatía)

Sesión 6. "Controlando mi ogro interior” (Autocontrol)

Sesión 7. "Queriéndonos mucho...” (Autoestima)

Sesión 8. "Lo positivo me hace bien” (Risoterapia)

Módulo del cuerpo bumano y desarrollo madurativo

Sesión 9. "Descubriendo el cuerpo humano I” (Partes del cuerpo)

Sesión 10. Ocupacional. "Descubriendo el cuerpo humano II” (Diferencias entre sexos)

Escolar. "Moviendo el cuerpito I” (Psicomotricidad)

Sesión 11. Ocupacional. "Como hemos cambiado...” (Cambios corporales a lo largo del ciclo vital)

Escolar. "Moviendo el cuerpito II” (Psicomotricidad)

\section{Módulo de habilidades afectivas}

Sesión 12. "Expresando afecto I” (Diferenciamos muestras de afecto)

Sesión 13. "Expresando afecto II" (Mural del afecto)

Módulo de habilidades sexuales - Grupo Escolar

Sesión 14. “¿¿Sexualiqué?!

Sesión 15. "Sumergiéndonos en el mar de la sexualidad"

Sesión 16. "Salimos a flote sanos y a salvo"

\section{Cierre y despedida}

Sesión 17: Fiesta de despedida y reconocimiento

Durante el proceso de implementación, se constató la necesidad de introducir modificaciones en la secuencia y temporalización de las sesiones. Por ejemplo, concretamente en el grupo del Centro Ocupacional, las sesiones de presentación fueron seguidas por las de habilidades sociales, para crear autoconfianza y cohesión grupal, fomentando así el área de inteligencia emocional. Tras esto, se centró la atención en el área cuerpo, conociendo sus partes y las diferencias entre mujeres y hombres. Uniéndose, por último, al contacto físico adecuado y a las diferentes formas de afecto que existen. Se decidió trabajar los aspectos sexuales durante todo el proceso, teniendo que prescindir del área abuso por falta de tiempo. Por lo tanto, no sólo se detectó la necesidad de modificar la estructura, sino también la temporalización de la intervención para adaptarla al ritmo de trabajo más pausado de estos hombres y mujeres con discapacidad intelectual. 


\section{Sistema y criterios de evaluación}

Se diseñaron cuestionarios para valorar la acción formativa tanto por los usuarios, como por los educadores (formadores y psicólogos) y sus familias, al inicio y al final de la intervención.

Los cuestionarios mantienen una estructura común. Comienzan con preguntas para evaluar el nivel de conocimiento y destreza en las diferentes áreas de la intervención, y concluyen con preguntas abiertas sobre expectativas hacia la intervención (al inicio) y valoración de la experiencia (al finalizar) (véase Anexo 1).

\section{Resultados}

Antes de la intervención, los tres agentes consideran que las personas que acuden al taller conocen las partes del cuerpo y las diferencias entre sexos, que tienen capacidad de relacionarse adecuadamente con los demás, que saben expresar afecto según contextos y relaciones, etc. En cambio, detectan escasos conocimientos en cuanto al placer corporal, prácticas sexuales, métodos anticonceptivos, etc.

Las expectativas preintervención hacen hincapié en el incremento en el bienestar social, personal y sexual de los chicos y chicas participantes. Ellos y ellas explicitan que les gustaría aprender tanto a conocerse mejor y mejorar su relación con sus compañeros y familias como diferentes aspectos sexuales importantes. Por otro lado, los educadores y familias manifiestan interés por conseguir reforzar la educación y autonomía de los chicos, y para que éstos adquieran conocimientos y destrezas adecuadas en sus relaciones afectivas y sexuales. También, les gustaría sentirse más tranquilos y mejorar su relación con ellos, a la vez que resolver dudas y pedir sugerencias.

Tras la experiencia, tal y como se refleja en las Figuras 1 y 2, se observan diferencias significativas en afectividad, sexualidad y habilidades sociales. Particularmente en el Centro Escolar, tras el análisis con la prueba T para muestras relacionadas del paquete estadístico SPSS, se registran cambios estadísticamente significativos ( $\mathrm{p}<$ .01) en la autopercepción del alumnado en las áreas de afectividad y sexualidad, y en la percepción de los educadores de mejoras en las habilidades sociales de sus alumnos. Mientras, en el Centro Ocupacional, también se registran cambios estadísticamente significativos $(\mathrm{p}<.01)$ en la autopercepción del alumnado en afectividad y sexualidad, y en la percepción tanto de educadores $(\mathrm{p}<.05)$ como de las familias $(\mathrm{p}<.01)$ en el área de la sexualidad. Esto es, las personas participantes puntúan más alto en cuestiones como el conocimiento de diferentes prácticas sexuales sanas y de prevención e incrementan su conocimiento en cuanto a las diferentes muestras de afecto que existen, potenciando las positivas y diferenciando entre contextos y relaciones. 


\section{Figura 1. Prueba-T, Centro-Escolar}

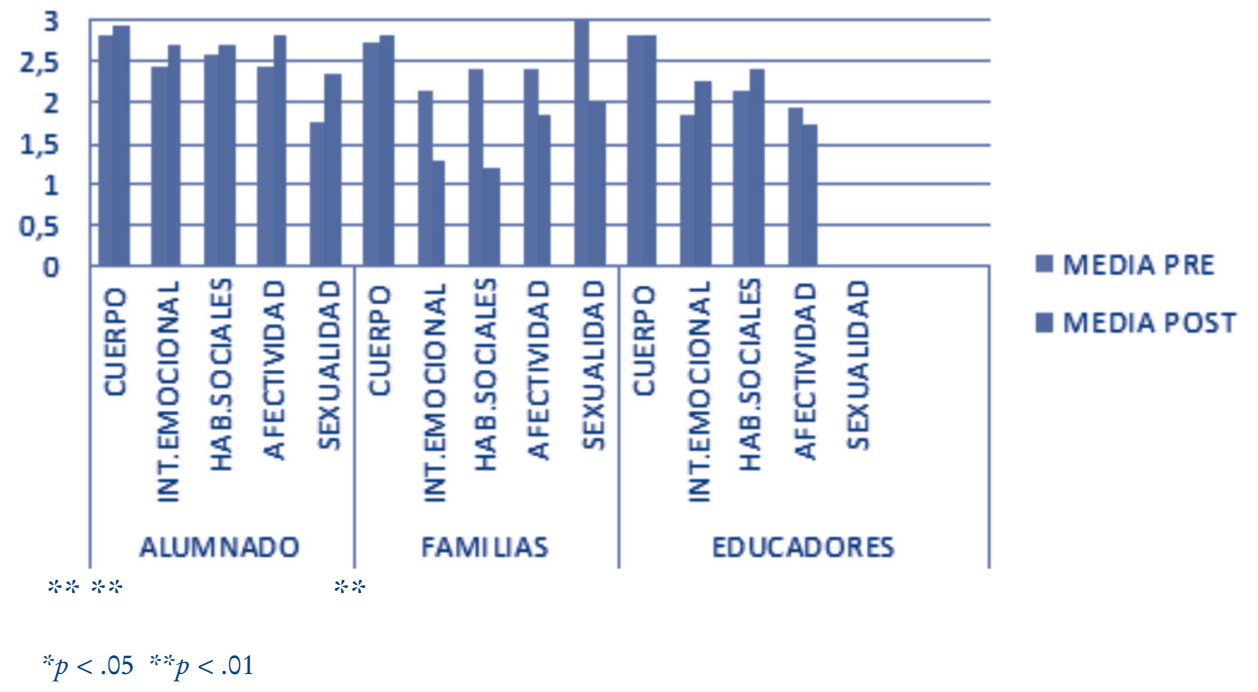

Figura 2. Prueba-T, Centro-Ocupacional

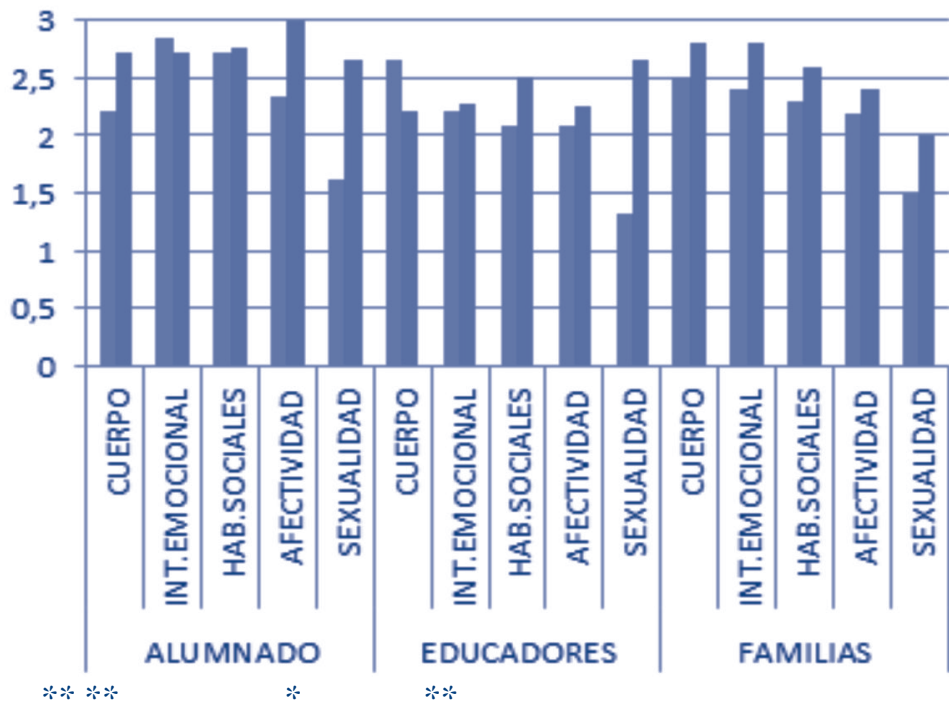

MEDIA PRE

MEDIA POST

$* p<.05 * p<.01$

(C) Ediciones Universidad de Salamanca

Siglo Cero, vol. 46 (4), n. ${ }^{\circ} 256,2015$, octubre-diciembre, pp. 45-58 
Las declaraciones valorativas aportan información relevante a la hora de dilucidar beneficios:

- “QQué tres cosas del taller te gustaron más?”:

- Los usuarios respondieron que lo que más les gustó, en primer lugar, fue el tocarse y abrazarse, a la vez que el bailar. En segundo lugar, destacan que les habían gustado tanto los monitores como el relajarse y el pintar.

- “¿Has notado cambios en los participantes?” y “¿En qué lo notas?”:

- Los educadores manifestaron beneficios en los participantes tanto en su educación afectivo-sexual, como en el incremento de la cohesión grupal, reflejada en expresiones como: "Han habido cambios y se nota en su forma de relacionarse" o "Se observa cohesión por su forma de compartir experiencias".

- Las familias consideraron que obtuvieron información suficiente sobre el programa, tanto por parte de los profesionales como a través de sus hijos e hijas. En cuanto a los cambios, explicitaron beneficios en sus hijos tanto a la hora de comunicarse (“Comentaba lo que hacían en el taller”), de reconocer su cuerpo y el de los demás ("Ahora conoce y habla más de las partes del cuerpo y de las diferencias entre hombres y mujeres”) y en cuanto a la afectividad (“Ahora es más cariñoso”).

- Psicólogos: “Ahora hablan de estos temas más abiertamente”.

- “¿Qué grado de satisfacción tiene ante el taller y los monitores?” y “¿Qué cambios observa en el grupo?":

- Tanto el psicólogo del Centro Escolar como la psicóloga del Centro Ocupacional mostraron una alta satisfacción con el taller y los monitores. Destacando tanto la capacidad de flexibilidad de los monitores como la motivación de los usuarios hacia el programa mediante la siguiente afirmación: "Han sabido ajustarse a las necesidades de nuestros/as usuarios/as y ello ha sido esencial para que fueran motivados al taller”. En cuanto a los cambios percibidos, remarcan el incremento de la cohesión grupal ("Ahora están más unidos”) y la comodidad para hablar de estos temas, que anteriormente les generaban rechazo (“Ahora hablan de estos temas más abiertamente”).

\section{Discusión y conclusiones}

Antes de la intervención, tanto los participantes como los educadores y sus familiares estimaban que los primeros conocían suficientemente su cuerpo, gozaban de habilidades sociales adecuadas y discriminaban entre contextos y relaciones. Si bien, en el área sexualidad, señalaban ciertas lagunas en el conocimiento de diferentes prácticas erótico-sexuales y preventivas.

Los contrastes entre la pre y la postevaluación arrojaron diferencias significativas en afectividad, habilidades sociales y sexualidad. Esto es, tanto usuarios como educadores y sus familias tienden a remarcar como los jóvenes que han participado en acción 
formativa muestran tras la intervención un mayor conocimiento sobre las diferentes prácticas erótico-sexuales y han desdramatizado el placer corporal. A su vez, conocen las manifestaciones de afecto, potencian las muestras positivas (abrazos, caricias...) y diferencian entre contextos (público/privado) y relaciones (amigos, conocidos, desconocidos). Estos resultados apuntan a que se ha conseguido dar respuesta a las demandas e intereses de los tres agentes implicados, incrementando así su bienestar personal, social y afectivo-sexual. En cualquier caso, estos resultados habrá que tomarlos con precaución dado que el limitado número de cuestionarios analizados supone un importante hándicap estadístico. Esta limitación se ve incrementada en el caso de las familias pues fueron muy pocas las que entregaron los cuestionarios pre y post cumplimentados.

Por último, las declaraciones valorativas aportaron información adicional relevante. Las personas que participaron explicitaron que del taller les gustó especialmente el contacto con sus iguales y monitores, el expresarse de manera creativa (bailar y pintar) y el disfrutar de las sensaciones corporales (relajación). Estas declaraciones explicitan la necesidad de potenciar la cohesión grupal, ya que genera bienestar y confianza, a la vez que favorece el aprendizaje. Los educadores manifestaron beneficios a nivel de cohesión grupal ("Han habido cambios en la forma de relacionarse”). Ello indica que la intervención favoreció la educación afectivo-sexual, a la vez que incrementó su desarrollo social, favoreciendo así la inclusión y normalización. Los familiares detectaron beneficios sobre todo a nivel social, tanto a la hora de expresarse como de relacionarse con los demás (“Ahora es más cariñoso”). Concuerda este dato con los cambios detectados por los psicólogos del centro, sobre el incremento de la cohesión grupal y la comodidad para hablar de estos temas tabú (“Ahora hablan más de estos temas que antes les generaban rechazo"). Estas valoraciones revelan que la intervención ha logrado incidir favorablemente en el bienestar general y desarrollo integral de los hombres y las mujeres con discapacidad intelectual.

Del estudio se extraen diferentes propuestas de mejora para futuras intervenciones. Una de las familias planteó la siguiente consideración: "Me parecería interesante una charla a la que acudieran los chicos, educadores y padres”. Esta mayor implicación familiar se considera un reto para futuras intervenciones, teniendo en cuenta que el contacto directo con ellas favorece la generalización de los beneficios.

El uso de cuestionarios como único instrumento de recogida de información revela ciertas limitaciones. Se propone enriquecer los instrumentos de evaluación con el uso de técnicas de corte cualitativo, como los grupos focales, y el registro situado de conductas claves, a cumplimentar por los monitores, equipo formativo y familia.

En esta primera ocasión, la intervención se ha llevado a cabo sólo en dos pequeños grupos. Pero, dada la satisfacción experimentada, el centro ya ha comenzado a propagarla al resto de sus usuarios.

\section{Referencias bibliográficas}

Alonso, M. y Aciego, R. (2003). Una experiencia de pintura expresiva y relajación con alumnos con necesidades educativas especiales. Cultura y Educación, 15 (2), 109-128. 
Barragán Medero, F. (1994). La educación sexual. Guía teórica y práctica. Barcelona: Ediciones Paidós Ibérica, S. A.

Bolaños, M. C., González, M. D., Jiménez, M., Ramos, M. E. y Rodríguez, M. I. (1994). Carpetas didácticas de educación afectivo-sexual. Tenerife: Consejería de Educación, Cultura y Deporte. Gobierno de Canarias.

Colectivo Harimaguada (2007). Sexpresan. Multimedia para la educación afectivo-sexual. Recuperado de http://www.harimaguada.org/sexpresan/.

García-Alonso, M. I. (2005). Concepto actual de discapacidad intelectual. Psychosocial Intervention, 14 (3), 255-276.

Ley Orgánica de Educación del 2/2006 de 3 de mayo. BOE número 106, de 4 de mayo de 2006.

Moretin, R., Rodríguez-Mayoral, J. M., Arias, B. y Aguado, A. M. (2006). Pautas para el desarrollo de programas eficaces de educación afectivo-sexual en personas con discapacidad intelectual. Siglo Cero: Revista Española sobre Discapacidad Intelectual, 37, 41-58.

Moretin, R., Verdugo, M. Á., Arias, B. y Rodríguez-Mayoral, J. M. (2006). Amor y enamoramiento en personas con discapacidad intelectual: un campo por explorar. Siglo Cero, 37, 59-81.

Navarro, Y., Torrico, E. y López, M. J. (2010). Programa de intervención psicosexual en personas con discapacidad. Educación y Diversidad, 4 (2), 75-92.

Rodríguez-Mayoral, J. M, Moretin, R., Arias, B. y López, F. (2006). Afectividad y sexualidad en personas con discapacidad intelectual: una propuesta de trabajo. Siglo Cero: Revista Española sobre Discapacidad Intelectual, 37 (217), 23-40.

Rubio, V. J. (1995). Las habilidades-sociales y la conducta adaptativa en el retraso mental. Competencia personal y competencia interpersonal. En M. Á. Verdugo (Ed.), Manual sobre personas con Discapacidad. Madrid: Siglo XXI.

WarricK, A. (2002). Comunicación sin habla. Comunicación Aumentativa y Alternativa alrededor del mundo. Madrid: CEAPAT.

\section{Agradecimientos}

El presente trabajo se realiza en el marco del Convenio de Colaboración de la Universidad de La Laguna y Hermanas Hospitalarias - Complejo ACAMÁN. Entidad, esta última, que tiene entre sus fines la prestación de servicios asistenciales, educativos $\mathrm{y}$ formativos a personas con discapacidad intelectual. 


\section{ANEXO 1 \\ Cuestionarios para educadores/as del Centro Ocupacional postintervención}

\section{CUESTIONARIO PARA RECABAR INFORMACIÓN SOBRE LOS RESULTADOS DEL PROGRAMA DE AFECTIVIDAD-SEXUALIDAD EN LOS ALUMNOS}

"Instrucciones para la realización del test"

Por favor, lea las afirmaciones y conteste relacionándolas con cada alumno/a.

- Para completar la primera parte del cuestionario, debe contestar marcando la opción que más se adecue al alumno/a en cuestión, ya sea Deficiente (-), Satisfactorio (+) o Consolidado (++).

- Si se equivoca tache o borre la respuesta incorrecta y marque la adecuada.

- En la segunda parte del cuestionario, debe responder de diferente manera, marcando tanto "SÍ” como "NO” según el ítem.

Nombre:

Fecha:

Indique el grado o nivel en que después del taller

\begin{tabular}{|c|c|c|c|c|c|c|}
\hline & 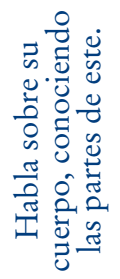 & 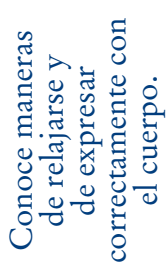 & 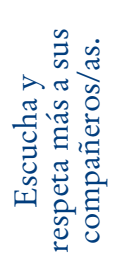 &  & 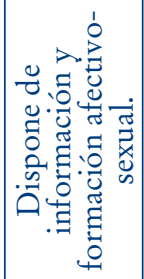 &  \\
\hline $\begin{array}{c}\text { Nombre } \\
\text { alumno/a } 1\end{array}$ & & & & & & \\
\hline $\begin{array}{c}\text { Nombre } \\
\text { alumno/a } 2(\ldots)\end{array}$ & & & & & & \\
\hline
\end{tabular}

(C) Ediciones Universidad de Salamanca

Siglo Cero, vol. 46 (4), n. ${ }^{\circ} 256,2015$, octubre-diciembre, pp. 45-58 
Creo que el trabajo llevado a cabo ha servido para:

\begin{tabular}{|l|l|l|}
\hline & SÍ & NO \\
\hline 1. Reforzar su educación y autonomía & & \\
\hline 2. Que los chicos/as tengan más conocimiento y formación en esta área & & \\
\hline 3. Mejorar mi relación con los alumnos/as & & \\
\hline 4. Estar más tranquilo/a respecto a mis alumnos/as & & \\
\hline 5. Que estos temas se traten solo en casa & & \\
\hline 6. Resolver dudas o pedir sugerencias & & \\
\hline
\end{tabular}

Considero que los avances logrados han sido fundamentalmente gracias a:

\begin{tabular}{|l|c|c|}
\hline & $S I ́$ & NO \\
\hline *El programa y los educadores & & \\
\hline El chico/a en cuestión & & \\
\hline El trabajo conjunto y coordinado & & \\
\hline La suerte & & \\
\hline
\end{tabular}

Antes de terminar, nos gustaría que nos ayudaras con una valoración personal sobre los logros obtenidos a nivel grupal. Responde "sí” o "no" y expón tu opinión donde se te indique.

1. ¿Les gustaba acudir al taller? ¿A quién crees que le gustaba más?

2. ¿Iban a gusto al taller?

3. ¿Salían contentos de las sesiones? ¿A quién notabas más contento/a?

4. ¿Se veían más unidos y cohesionados? ¿En qué lo notas?

5. ¿Has notado otros cambios después del taller en los chicos/as? ¿De qué cambios se trata? ¿A quién crees que le sirvió más? 
En el siguiente espacio podrás escribir cualquier comentario que consideres oportuno:



¡Muchas gracias por contestar! ¡Nos ha encantado contar contigo! 